Nereis. Revista Iberoamericana Interdisciplinar de Métodos, Modelización y Simulación

\title{
Drugs Repurposing for Coronavirus Treatment: Computational Study Based On Molecular Topology
}

Readaptación de fármacos en el tratamiento contra el coronavirus. Estudio computacional basado en topología molecular

Fecha de recepción y aceptación: 23 de marzo de 2020, 23 de marzo de 2020

DOI: $10.46583 /$ nereis_2020.12.591

\author{
Jorge Gálvez ${ }^{1^{*}}$, Riccardo Zanni ${ }^{1,2}$ y María Gálvez-Llompart ${ }^{1,3}$ \\ ${ }^{1}$ Unidad de Diseño de Fármacos y Topología Molecular. Departamento de Química Física. Universidad de Valencia. \\ ${ }^{2}$ Unidad de Microbiología y Patología de Plantas (CSIC-UMA). Departamento de Microbiología. Facultad de Ciencias. Universidad \\ de Málaga. \\ ${ }^{3}$ Instituto de Tecnología Química (UPV-CSIC). Universidad Politécnica de Valencia. \\ ${ }^{*}$ Correspondencia: Universitat de València. Facultad de Farmacia. Av. Vicent Andres Estellés s/n. 46100 (Burjassot), Valencia. \\ España.E-mail: jorge.galvez@uv.es
}

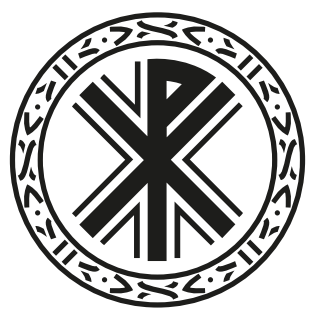

KEYWORDS: coronavirus, COVID-19, new drugs, drugs repurposing, molecular topology.

PALABRAS CLAVE: coronavirus, COVID-19, nuevos tratamientos, reposicionamiento de fármacos, topología molecular.

\section{SUMMARY}

The present communication illustrates the results of a computational study based on molecular topology, focused on the repositioning of drugs to treat the SARS-CoV-2 virus, better known as coronavirus, responsible for the COVID-19 disease. Using lopinavir, a well-known viral protease inhibitor as the reference drug, a mathematical pattern is found allowing the screening of the market drugs, searching for potential candidates to inhibit the said enzyme. This way new possible therapeutic alternatives to fight the coronavirus are found.

Results indicate that antivirals such as brecanavir, as well as various groups of drugs, among which are antibiotics of the macrolide family (azithromycin, clarithromycin and erythromycin among others) could be useful in treating COVID-19 infection. 


\section{RESULTS AND DISCUSSION}

The Molecular Topology and Drug Design Research Unit from the University of Valencia (Spain) has been working in a pioneering way since the 1980s in designing and selecting new drugs based on a paradigm called Molecular Topology (MT). Some of these new drugs have been internationally patented in fields as diverse as cancer, Alzheimer's and malaria [1].

In essence, the MT method consists in characterizing the molecules by a set of numbers, called topological indices, which basically depend on the structure of each molecule and therefore on its pharmacological activity. In this way, it is possible to group the molecules into sets that respond to different mathematical patterns according to their activity. For example, we can have the anticancer pattern, even the anticancer pattern associated with a specific mechanism (for example, topoisomerase inhibitors).

Once a mathematical pattern has been found, it is easy to ask the computer to search for new molecules matching the same pattern and therefore showing the same pharmacological activity, but with better pharmacodynamic, pharmacokinetic, or toxicological properties (more potency, better absorption, less toxicity, etc.). By using a mathematical pattern to characterize pharmacological activity, the authors are able to identify a great structural variability within the potential active compounds.

Given the current pandemic situation, now that the structure of the virus protease has been published [2] and that some antivirals inhibiting proteases have been published as slightly effective against the virus, the authors in this communication disclose the results of a topological screening of the market drugs, selecting those which may present significant anti-COVID-19 activity.

One of them, which we have taken as the gold-standard or reference drug, is lopinavir, which according to various publications seems to have shown effectiveness against SARS-CoV-2.

After obtaining their topological-mathematical fingerprint through seven predictive equations (not reflected here), the authors proceeded to screen two databases of pharmacological compounds: Comprehensive Medicinal Chemistry [3] and DrugBank [4], altogether some 15,000 molecules.

Table 1. Results obtained from the virtual screening of selected drugs as potential protease inhibitors and therefore useful in the treatment of COVID-19

\begin{tabular}{ccc}
\hline Drug & Family & Inhibitory capability to SARS- CoV-2 protease \\
\hline Nonactine & Antibiotic & 10,0 \\
Diproleandomycin & Antibiotic & 8,3 \\
Flurithromycin & Antibiotic & 7,3 \\
Brecanavir & Antiviral & 7,3 \\
Clarithromycin & Antibiotic & 7,0 \\
Erythromycin & Antibiotic & 6,9 \\
Lexithromycin & Antibiotic & 6,8 \\
Ritonavir & Antiviral & 6,8 \\
Argadin & Others & 6,4 \\
Azithromycin & Antibiotic & 6,1
\end{tabular}




\begin{tabular}{ccc}
\hline Drug & Family & Inhibitory capability to SARS- CoV-2 protease \\
\hline Cethromycin & Antibiotic & 5,7 \\
Oxidized Glutathione & Antineoplastic & 5,6 \\
Fomidacillin & Antibiotic & 5,4 \\
Neutramycin & Antibiotic & 5,1 \\
Murabutide & Immunostimulant & 5,0 \\
Mirosamicin & Antibiotic & 4,8 \\
Cronidipine & Calcium channels' blocker & 4,5 \\
Cefclidin & Antibiotic & 4,1 \\
Temurtide & Antiviral & 4,0 \\
Cefuroxime & Antibiotic & 3,9 \\
Pentigetide & Antialergic & 3,9 \\
Cistinexine & Expectorant and Mucolytic & 3,6 \\
Cefbuperazone & Antibiotic & 3,2 \\
Lopinavir & Antiviral & 2,9 \\
\hline
\end{tabular}

The right column indicates the range of inhibitory potency against protease of the different drugs with an index (in arbitrary units). The reference drug lopinavir appears in bold at the bottom and showed a value of 2.9 on this scale.

As can be seen, there is a surprisingly high number of drugs that show theoretical activity against the virus protease, including known antivirals, antibiotics of different types and other groups of drugs (antineoplastic, antiallergic, etc.).

Among the most active are brecanavir (potency index 7.3) and an ionophore antibiotic, nonactin (index 10, the maximum). It is also noteworthy that there are some antibiotics from the macrolide family, such as clarithromycin and erythromycin. Another potentially useful drug is murabutide, since it is a known immunostimulant, which could promote a synergy.

Obviously, only experimental confirmation of the activity in vitro and in vivo of these compounds against the virus will demonstrate their real efficacy. However, the repositioning of drugs can shorten significantly the time in providing therapeutic alternatives to deal with the current public health crisis. In this sense, the authors believe that the drugs reported in Table 1 represent an important contribution, whose fast achievement is another proof of MT's power as a tool for the search of new drugs.

\section{ACKNOWLEDGMENT}

The authors thank Dr. D. Juan Martínez Hernández for his support to our research group and his willingness to collaborate in joint projects of social and health interest. 


\section{LITERATURE CITED}

[1] Galvez J. Molecular Topology and Drug Design Unit. Jorge Galvez Researchgate profile page. Available at: https://www.researchgate.net/profile/Jorge_Galvez2.

[2] The Protein Data Bank archive (PDB). New Coronavirus Protease Structure Available. Available at: http://www.wwpdb.org/news/news\#5e39e03fa5007a04a313edc3.

[3] CMC-database. Comprehensive Medicinal Chemistry. Available at: http://www.akosgmbh.de/ accelrys/databases/cmc-3d.htm.

[4] DrugBank. Available at: https://www.drugbank.ca/. 